\title{
R EVUE
}

\section{INTERNATIONA LE}

DE LA

CROIX-ROUGE

ET

\section{BULLETIN INTERNATIONAL}

DES

SOCIÉTÉS DE LA CROIX-ROUGE

inter arma

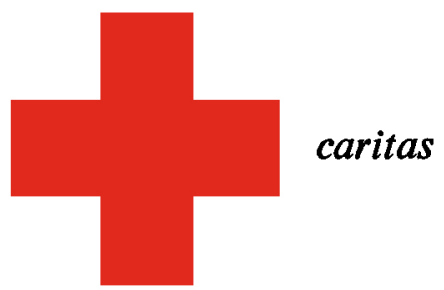

COMITÉ INTERNATIONAL DE LA CROIX-ROUGE GENÈVE 


\section{COMITÉ INTERNATIONAL DE LA CROIX-ROUGE}

\section{à Genève}

MM. MAX HUBER, docteur en droit, ancien président de la Cour permanente de justice internationale président d'honneur (1923) ${ }^{1}$

PAUL RUEGGER, docteur en droit, ancien ministre de Suisse en Italie et en Grande-Bretagne, président (I048)

JACQUES CHENEVIERE, docteur ès lettres h. c. (I9I9)

M"10 LUCIE ODIER, ex-chef du Service des infirmières-visiteuses de la Section genevoise de la CroixRouge suisse (1930)

MM. CARL J. BURCKHARDT, docteur en philosophie, ancien ministre de Suisse en France (1933) EDOUARD CHAPUISAT, docteur ès lettres h. c. (I938)

ALEC CRAMFR, docteur en médecine, colonel (1938)

MARTIN BODMER, docteur en philosophie h. c. (1940)

ERNEST GLOOR, docteur en médecine, vice-président (1945)

RENÉ VAN BEIRCHEM, docteur en droit, banquier (I946)

LEOPOLD BOISSIER, docteur en droit, professeur à l'Université de Genève, ancien secrétaire général de l'Union interparlementaire $(\mathrm{r} 9+6)$

PAUL CARRY, docteur en droit, professeur à l'Université de Genève (I946)

EDMOND GRASSET, docteur en médecine, professeur à l'Université de Genève, directeur de l'Institut d'hygiène (1940)

HENRI GUISAN, généra], ancien commandant en chef de l'armée suisse (1948)

ALFREDO VANNOTTI, docteur en médecine, professeur a l'Université de Lausanne (1949)

RODOLFO OLGIATI, ancien directeur du Don suisse (1949)

MHe MARGUERITE VAN BERCHEM (I95I)

MM. FRÉDÉRIC SIORDET, avocat, conseiller du Comité international de la Croix-Rouge depuis I943, vice-président (I95I)

MARCEL JUNOD, docteur en médecine, délégué du Comité international de la Croix-Rouge de I935 à 1946 (I952)

ERNEST NOBS, ancien conseiller fédéral (1952)

\section{Direction :}

MM. ROGER GALLOPIN, directeur exécutif

JEAN S. PICTET, directeur des Affaires générales

DAVID DE TRAZ, directeur exécutif-adjoint

EDOUARD DE BONDELI, sous-directeur

CLAUDE PILLOUD, sous-directeur des Affaires générales

Secrétariat général:

M. JEAN DUCHOSAL, secrétaire général

1 Les années indiguées dans les parenthèses désignent tes dates de nomination des membres du Comité international. 


\section{REVUE INTERNATIONALE DE LA CROIX-ROUGE}

TRENTE-SIXIEME ANNEE - N० 432

DECEMBRE 1954

SOMMAIRE

COMITÉ INTERNATIONAL DE LA CROIX-ROUGE

Réunion des représentants de Sociétés nationales (11 novembre

1954). - Liste des représentants . . . . . . . . . 961

La position de l'individu dans l'ordre juridique international, par Maurice Bourquin, professeur à l'Université de Genève et à 1'Institut universitaire de Hautes études internationales (suite et fin)

Limites de l'intégration des réfugiés en matière de droit privé, par Murad Ferid, professeur, directeur de l'Institut de droit comparé de l'Université de Munich. . . . . . . . . .

\section{PROTECTION DES POPULATIONS CIVILES}

Réunion des représentants de Sociétés nationales : Exposé introductif de M. Frédéric Siordet, vice-président du Comité international de la Croix-Rouge ..........

\section{A TRAVERS LES REVUES \\ * \\ BULLETIN INTERNATIONAL DES SOCIÉTÉS DE LA CROIX-ROUGE}

(Voir sommaire, page 1021)

Parait à la fin de chaque mois

Edrreur : Comité international de la Croix-Rouge

RÉDACTEUR RESPONSABLe : Louis Demolis

Prox, franco, un an : Fr. 20.--; le numéro Fr. 2.-. Administration et publicité : CICR, 7, av. de la Paix, Genève. Téléphone 3330 60. Compte de chèques I.1767 
SOMMAIRE

\section{EN LANGUE ANGLAISE}

December 1954, No. 12, Vol. VII :

International Committee of the Red Cross : Recent Activities. - Red Cross and the Menace of Total Warfare.

The Slave Question, by Henri CoURSIER, Member of the Legal Service of the ICRC (continued).

Table of Contents. Vol. VII (1954).

$*$

\section{EN LANGUE ESPAGNOLE}

Diciembre 1954, No 12, Vol. VI :

Comité Internacional de la Cruz Roja : Actividades recientes. - Guerra atómica, química y bacteriológica. Protección de las Poblaciones civiles.

Indice de Sumarios. Vol. VI (1954).

\section{$*$ \\ EN LANGUE ALLEMANDE \\ Dezember 1954, Nr. 12, Band V:}

Internationales Komitee vom Roten Kreuz : Die Tätigkeit des IKRK in der letzten Zeit. - Das Rote Kreuz und die Drohung des totalen Krieges.

Der Internationale Flüchtlingsschutz, von Paul WEIS, Rechtsberater des Hohen Kommissariates des Vereinten Nationen für Flüchtlinge (Fortsetzung und Schluss).

Inhaltsverzeichnis. Band V (1954). 


\section{Votre Banque...}

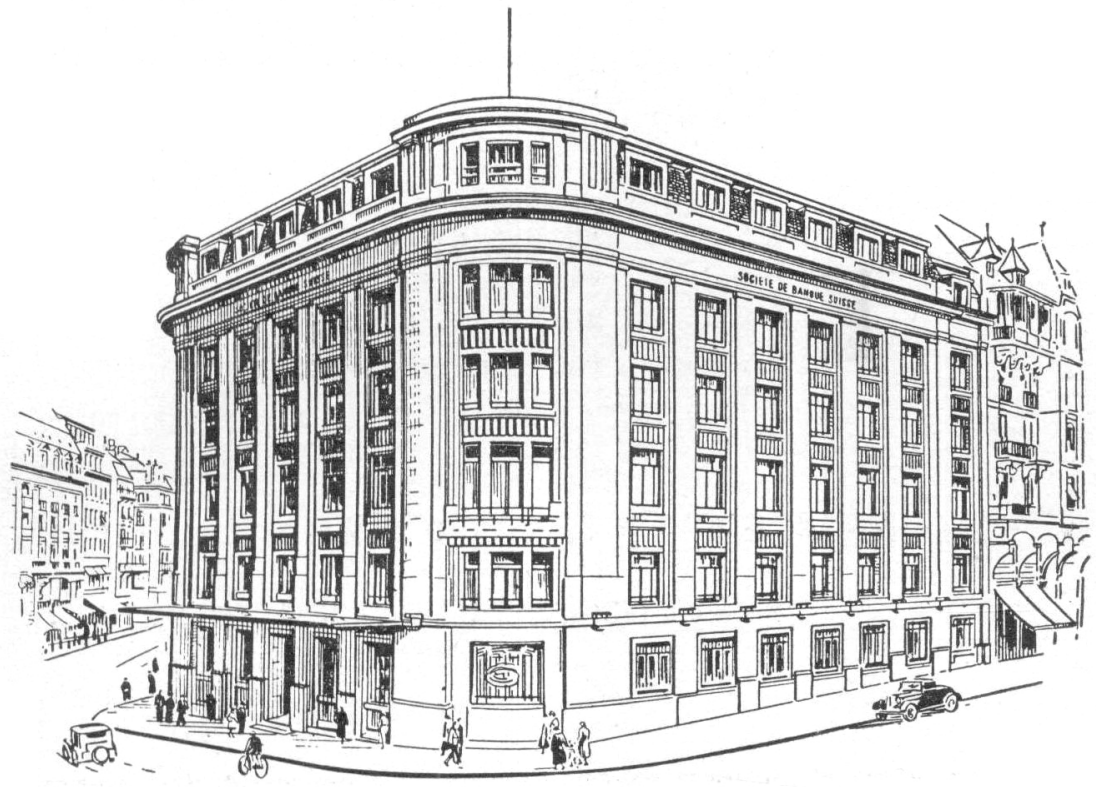

\section{LA SOCIÉTÉ DE

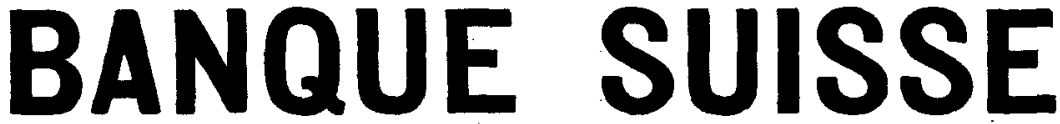 GENEVE}

2, rue de la Confederation

BALE ZURIOH ST-GALL LAUSANNE NEUCHATEL CHAUX-DE-FONDS SCHAFFHOUSE BIENNE LONDRES NEW-YORK

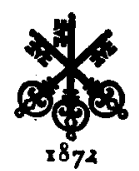

CAPITAL ET RÉSERVES FRS 226 MILLIONS 


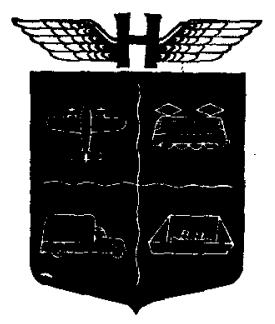

Compagnie d'Assurances Générales

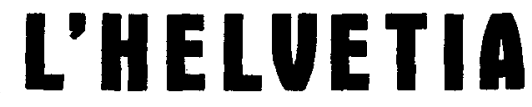

ST-GALL (Suisse)

Fondée en 1858

$\begin{array}{lc}\text { Capital social ( } 1 / 2 \text { versé) } & \text { Francs suisses } \\ \text { Total des moyens de garantie } & 61.900 .00000\end{array}$

\title{
ASSURAMGES TRAHSPORTS
}

REAssuRahices aans toutes les Branches

\author{
Direction: ST-GALL (Suisse) Tólóphone: (071) 233121 \\ TElégrammos : HELVETIA ThANSPORT
}

Agences en Suisse et dans beaucoup d'autres pays 


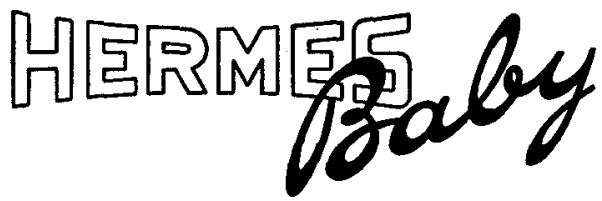

\section{LA PREMIÈRE DES PORTATIVES · LÉGÈRES DEPUIS 1935}

Le dernier modèle

comporte 20 dispositifs

et perfectionnements nouveaux!

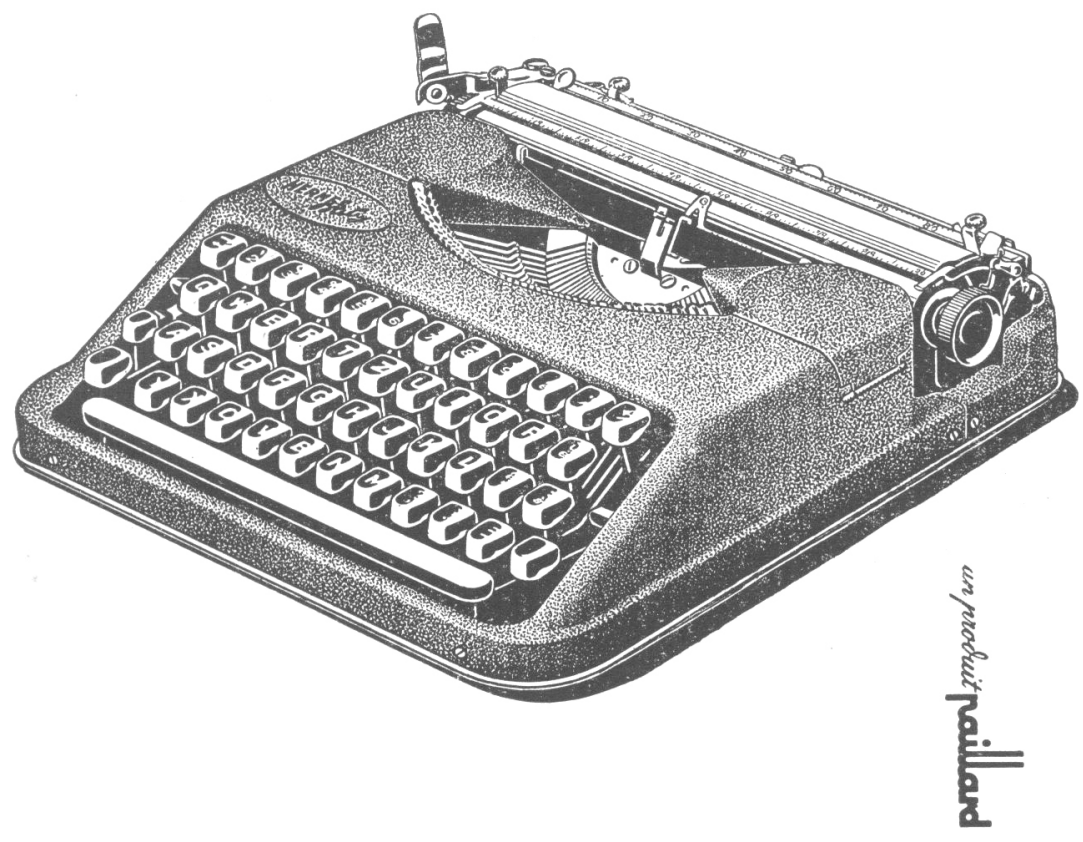

PAILLARD S.A. YVERDON/SUISSE (Fondée en 1814) 


\section{SOCIÉTÉ FIDUCIAIRE ROMANDE OFOR S.A.}

- Revisions et organisations comptables

- Administration et liquidation de sociétés

- IMPOTS : Conseils par spécialiste

9, RUE D'ITALIE - GENEVE TELEPHONE 2432 81/3
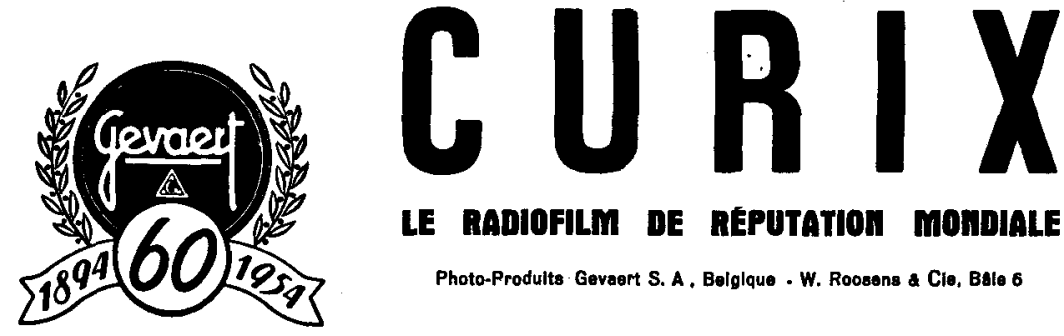

\section{LE RADIOFILM DE REPUTATIOH MOHDIALE}

Photo-Prodults Gevaert S. A. Belglque - W. Roosens a Cie, Bale 6
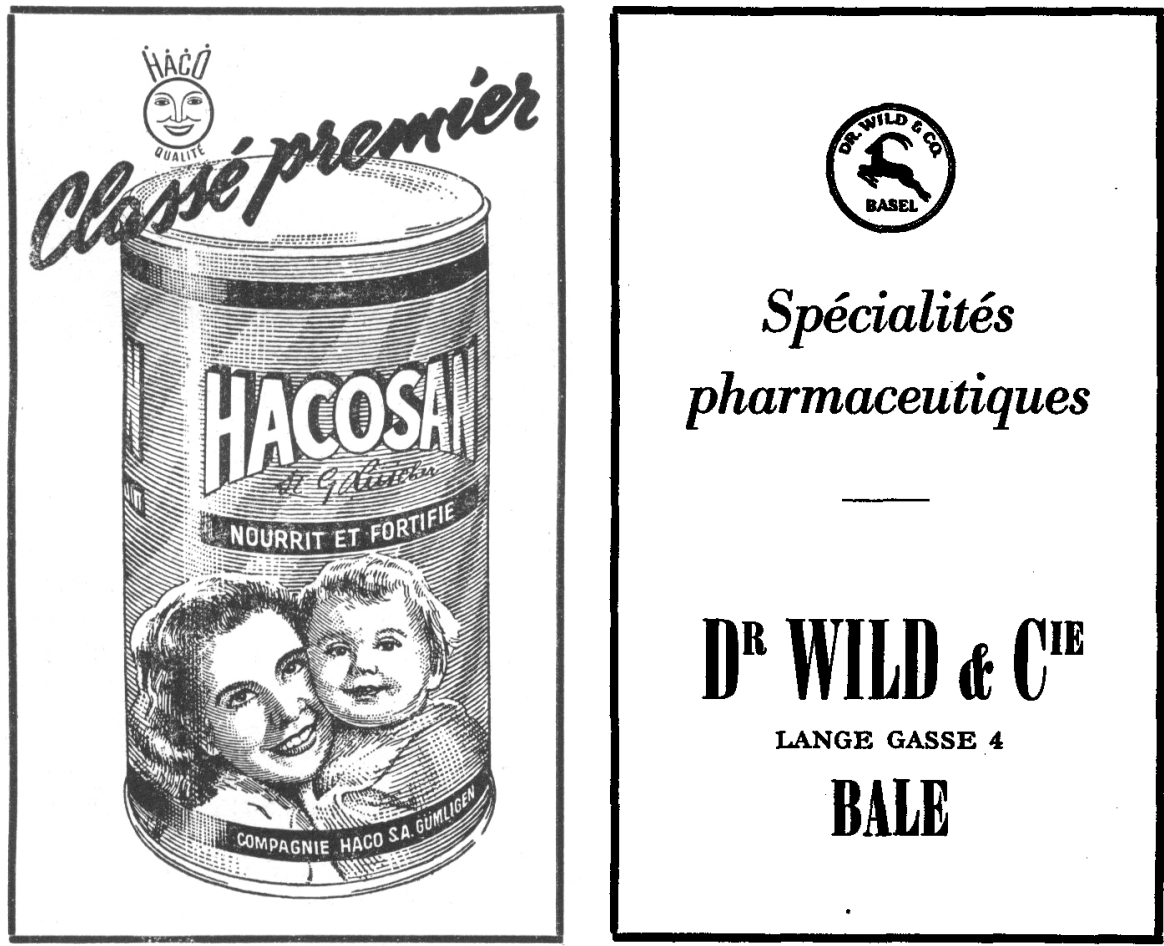

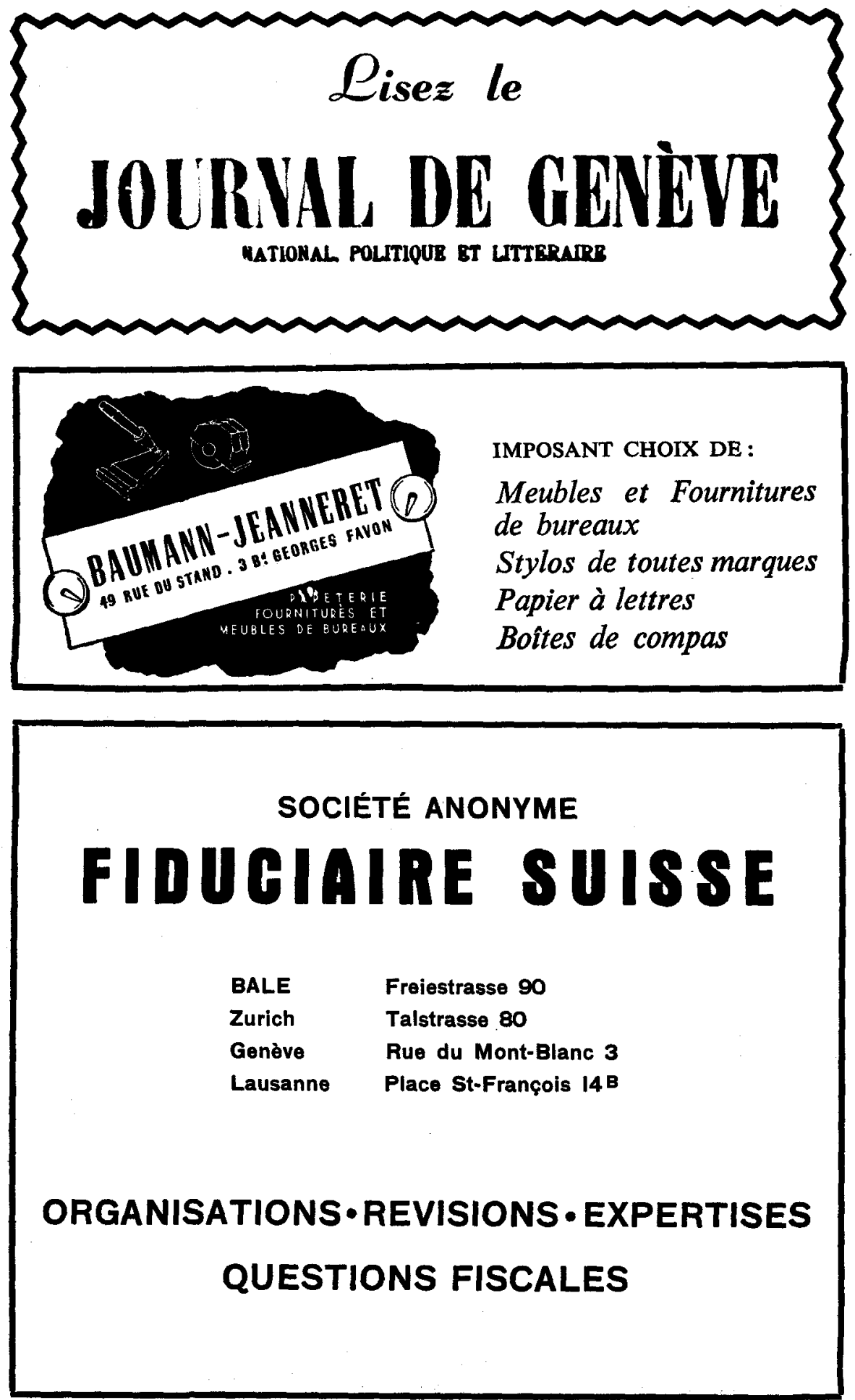


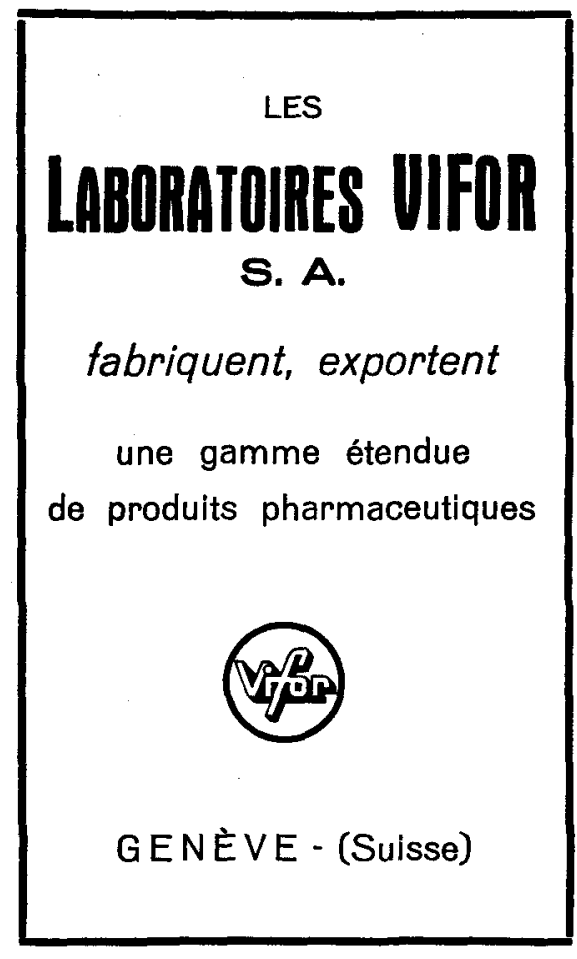

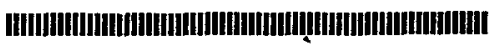

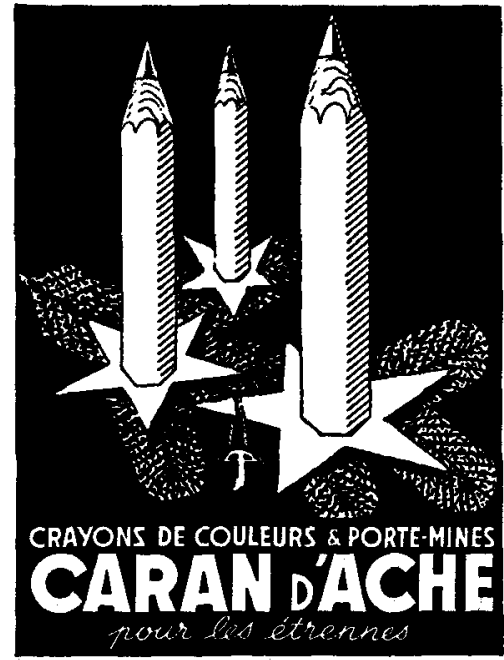

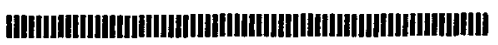

\section{HUGUENIN}

Médailleurs defuis 1868

\section{LE LOCLE} Suls

Hraignes, Nlédaitles, LPaquettes en exécution artiabique
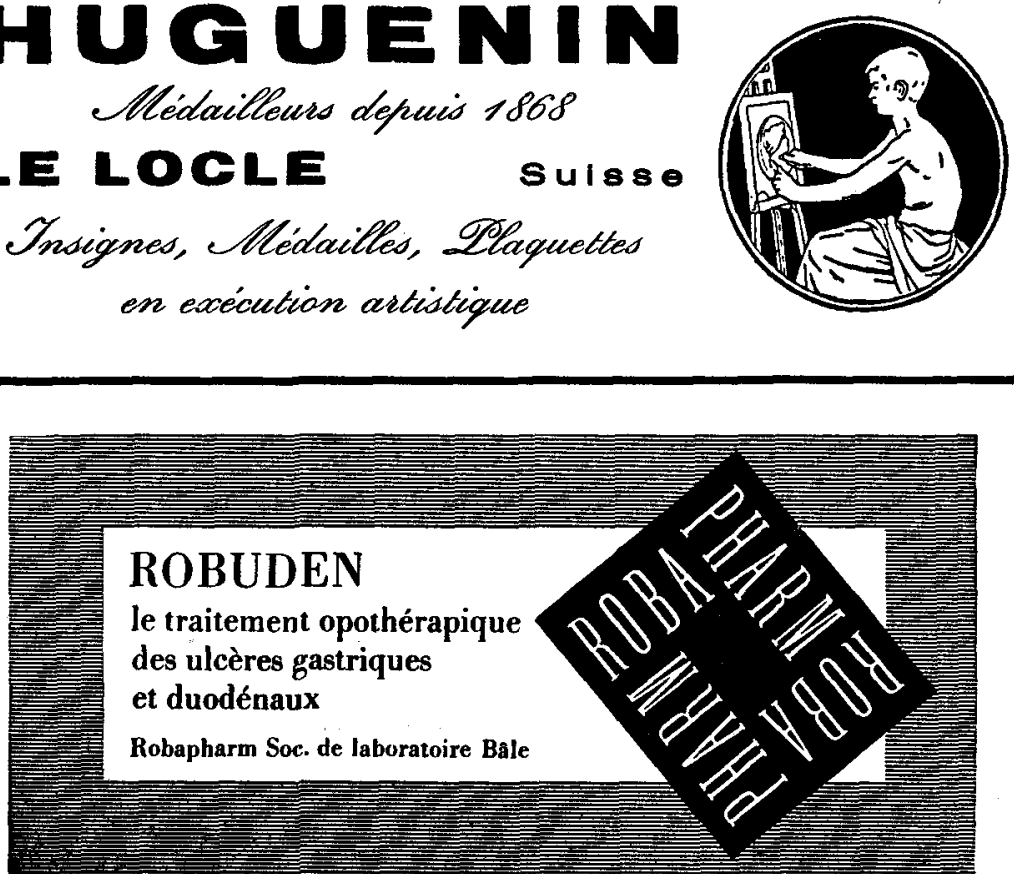


\section{TRAMSPORTS IHTERMATIOHAUX}

\section{PASSAGES - VOYAGES}

Waisen fondte en 1815

Soclete par Actions

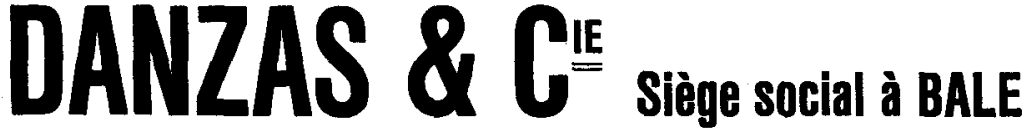

Succursales a GEN EVE, Gare Voyageurs-Cornavin,

Bienne, Brigue, Buchs, Chlasso, Lucerne, Schaffhouse, St-Gall, St-Margrethen, Vallorbe, Zurich.

Parls, 15, rue de Nancy (10'), Annemasse, Belfort, Bellegarde, Besançon, Colmar, Delle, Modane, Mulhouse, Nancy, Pontarller, Strasbourg.

Bureaux de voyages à GENEVE, rue du Mont-Blanc No 5 ,

Băle, Blenne, Buchs, Lugano, St-Gall, Schaffhouse, Zurich.

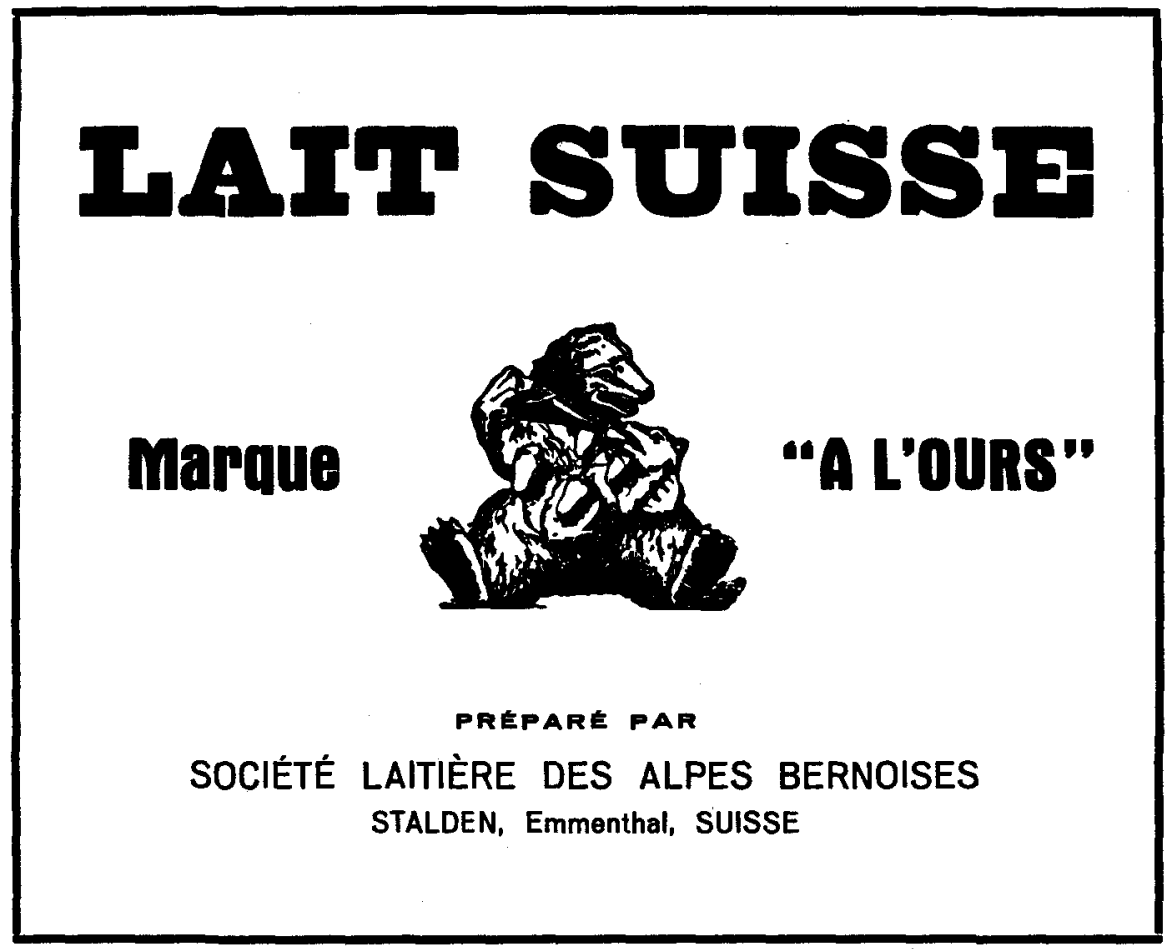



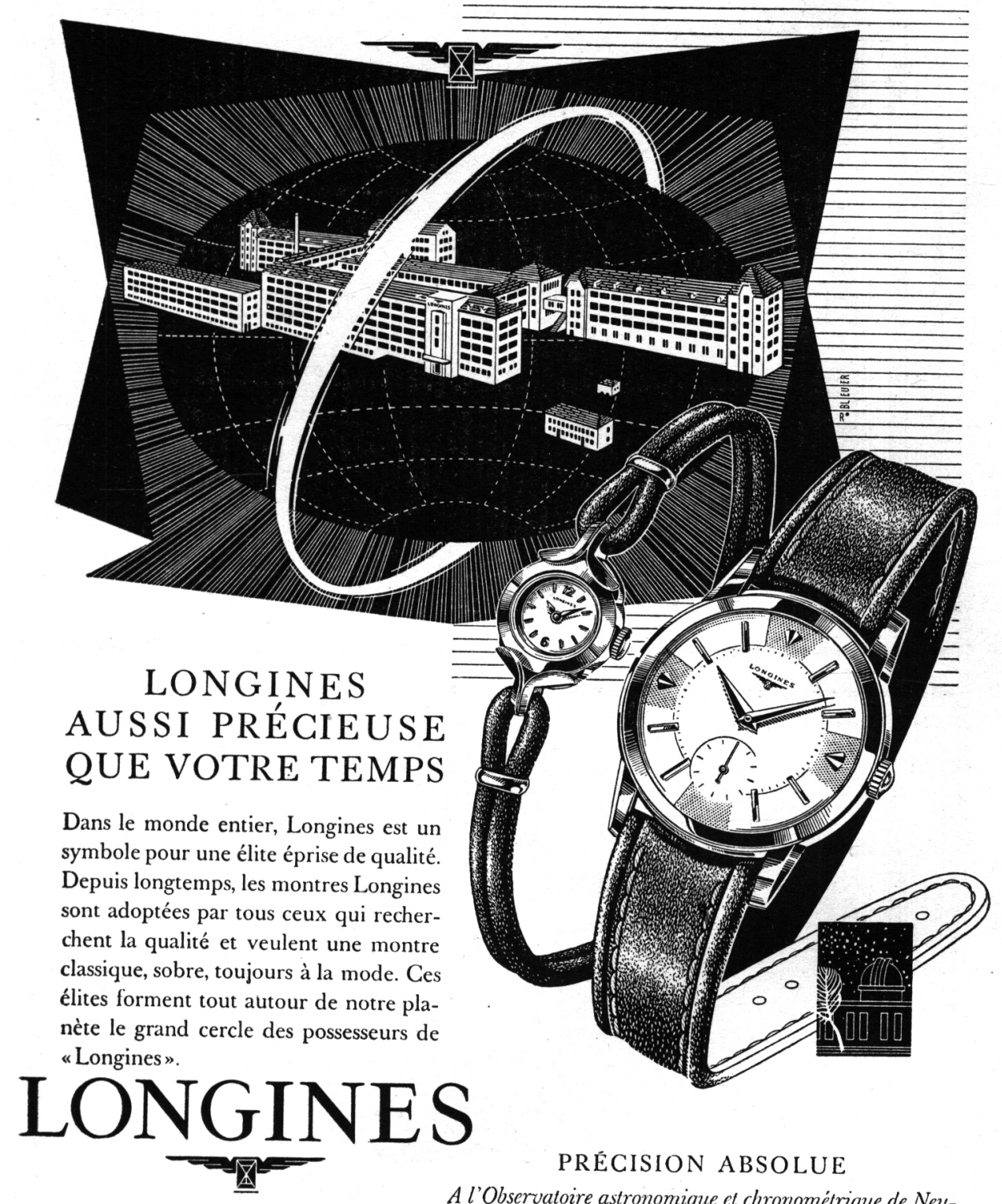

\section{PRÉCISION ABSOLUE}

A l'Observatoire astronomique et chronométrique de Neuchâtel, la "Chronocinégines Longines", qui permet de mesurer un temps au centième de seconde avec la possibilité d'apprécier les millièmes, n'accuse aucune erreur de marche après 24 heures de fonctionnement.

Aucun instrument horaire transportable n'a jamais atteint parcil résullat. 


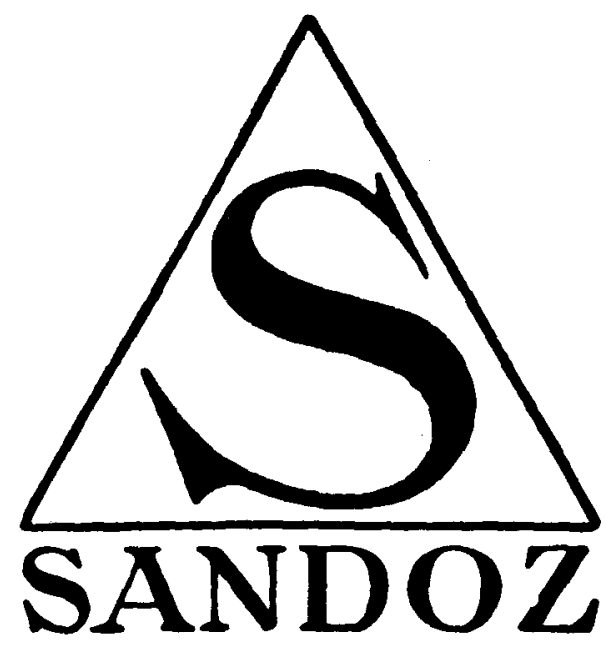

PRODUITS

PHARMACEUTIQUES

BALE (Suisse) 


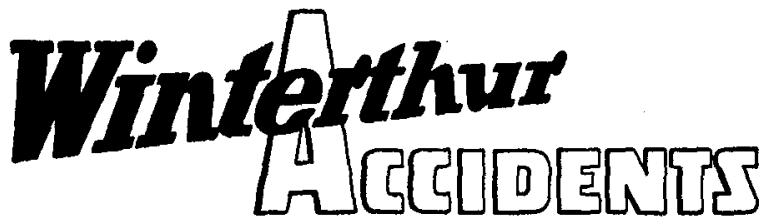

Société Suisse d'Assurance contre les Accidents à Winterthur

\section{Assurances Accidents,} Responsabilité civile, Automobiles, Vol et Cautionnements

à des conditions avantageuses 